\title{
A interventional study in a real life setting to assess the clinical efficacy and effect to fracture in the 1 year after the injection of zoledronic acid in osteoporotic patients with long bone or spine, pelvic fractures
}

Jaewon Lee, Joonguk Kim, Ki-chul Park, Ye-Soo Park

Department of Orthopedic Surgery, Guri Hospital, Hanyang University

College of Medicine, Guri city, Gyeonggi-do, Korea 


\section{Introduction}

\section{Zoledronate}

- Oral bisphosphonate has gastrointestinal side effect, and low compliance. Zoledronate provide an alternative for patients $w$ ho cannot tolerate oral bisphosphonate.

* HORIZON-PFT trial (7765 postmenopausal women)

Once-yearly infusion of zoledronic acid during 3 year significa ntly reduced the incidence of vertebral, hip, and non-vertebra I fracture. 


\section{Introduction}

To investigate

- The change in bone mineral density (BMD)

- Change in back pain (VAS)

- Fracture healing

- Newly developed fracture

$\checkmark$ Adverse effect

in elderly postmenopausal osteoporosis patients who has had ver tebral fracture or non-vertebral fracture treated with zoledronate 


\section{1. - 2012. 06.}

-Postmenopausal women who had osteoporosis (T score <-2.5)

-with osteoporotic vertebral fracture or non-vertebral fracture -who approved the informed consent

Zoledronate injection

-IV zoledronate (Aclasta $\left.{ }^{\circledR}, 5 \mathrm{mg} / 100 \mathrm{ml}\right)$

-1 time injection

-3 days after the fracture diagnosed
Exclusion: patients who has contraindications of IV zoledronate fracture not related with osteoporosis

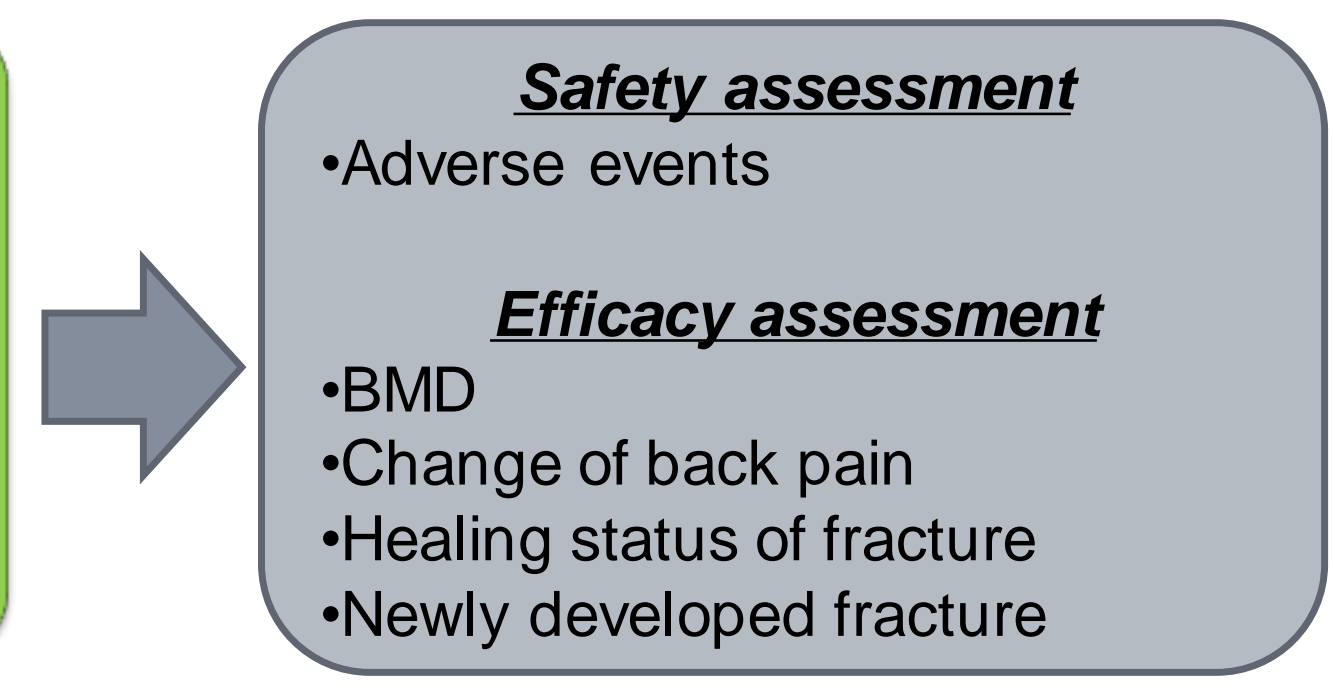




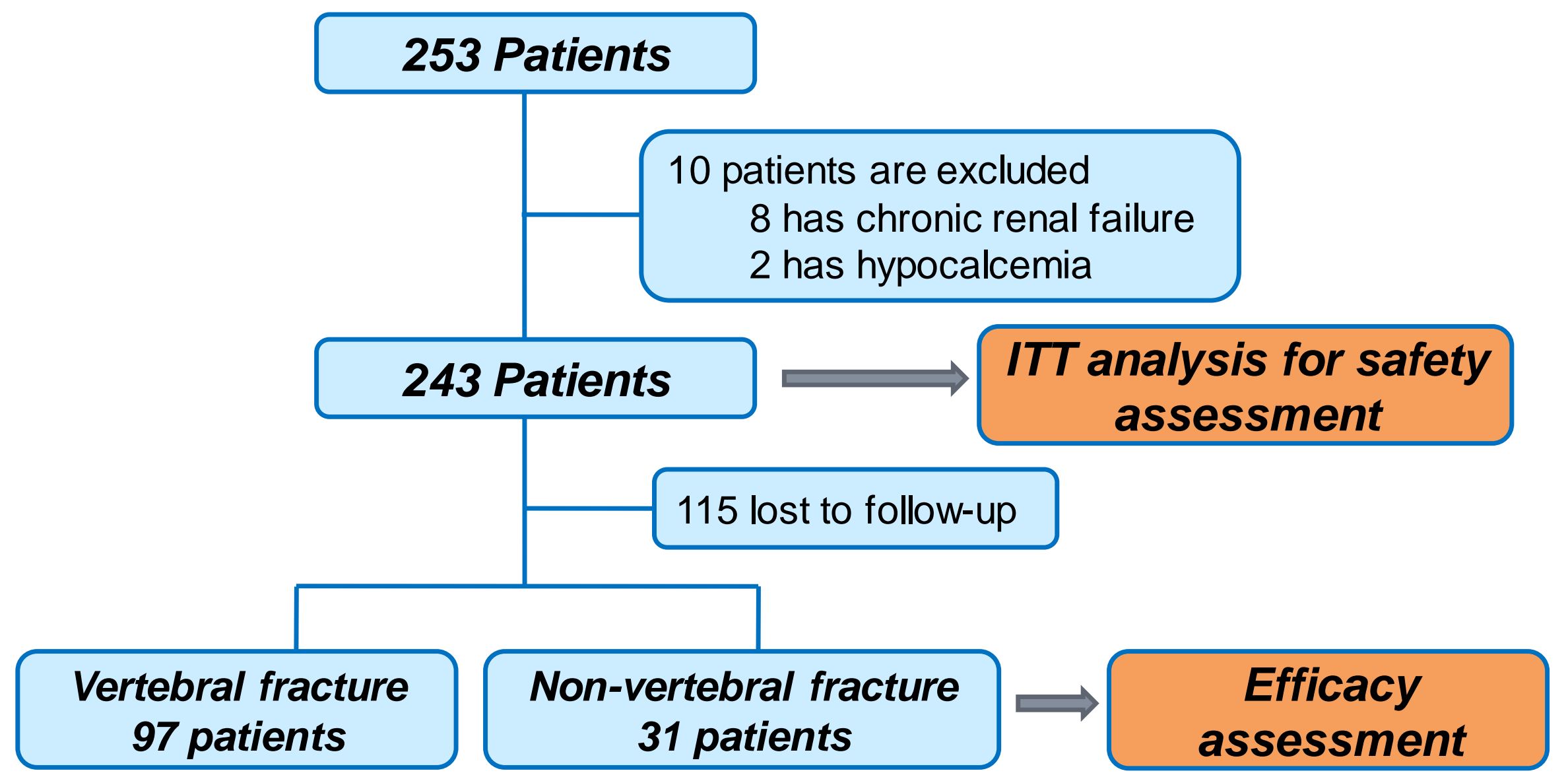




\section{Study groups $(N=130)$}

Mean age $(y r s)=.71.1 \pm 9.1$

\begin{tabular}{|c|c|c|c|}
\hline & Baseline & $\begin{array}{c}\text { 12 months after } \\
\text { Zoledronate injection }\end{array}$ & \begin{tabular}{l} 
P-value \\
\hline Vertebra $\boldsymbol{T}$-score
\end{tabular} \\
\hline Femoral neck $\boldsymbol{T}$-score & $-3.00 \pm 0.77$ & $-2.70 \pm 1.47$ & $P<0.05$ \\
\hline Lowest $\boldsymbol{T}$-score & $-2.80 \pm 0.77$ & $-2.70 \pm 0.87$ & $P<0.05$ \\
\hline
\end{tabular}




\section{Vertebral fracture group ( $N=97)$}

Mean age $(y r s)=.71.9 \pm 9.4$

\begin{tabular}{|c|c|c|c|}
\hline & Baseline & $\begin{array}{c}12 \text { months after } \\
\text { Zoledronate injection }\end{array}$ & \begin{tabular}{l} 
P-value \\
\hline Vertebra $\boldsymbol{T}$-score
\end{tabular} \\
\hline Femoral neck $\boldsymbol{T}$-score & $-3.14 \pm 0.85$ & $-2.76 \pm 1.61$ & $P<0.05$ \\
\hline Lowest $\boldsymbol{T}$-score & $-2.92 \pm 0.84$ & $-2.76 \pm 1.09$ & $P<0.05$ \\
\hline
\end{tabular}




\section{Non-vertebral fracture group $(N=31)$}

Mean age (yrs.) $=70.3 \pm 8.5$

\begin{tabular}{|c|c|c|c|}
\hline & Baseline & $\begin{array}{c}12 \text { months after } \\
\text { zoledronate injection }\end{array}$ & \begin{tabular}{l} 
P-value \\
\hline Vertebra $\boldsymbol{T}$-score
\end{tabular} \\
\hline Femoral neck $\boldsymbol{T}$-score & $-2.85 \pm 1.03$ & $-2.71 \pm 0.84$ & $P<0.05$ \\
\hline Lowest $\boldsymbol{T}$-score & $-2.71 \pm 0.61$ & $-2.66 \pm 1.01$ & $P<0.05$ \\
\hline
\end{tabular}


* Delayed healing, Nonunion : 0 case

- New developed fracture : 3 cases

$\checkmark$ Vertebral fracture : 2 cases

$\checkmark$ Distal radius fracture : 1 case 
* Reported side effects : 11 cases (4.5\%)

$\checkmark$ Flu-like symptom : 6 cases

$\checkmark$ Nausea/vomiting : 1 case

$\checkmark$ Dyspepsia: 1 case

$\checkmark$ Joint swelling : 1 case

$\checkmark$ Arthralgia : 1 case

$\checkmark$ Dizziness : 1 case 
* Administration of zoledronate in postmenopausal osteoporosis patient who had vertebral fracture or non-vertebral fracture

$\checkmark$ Bone mineral density improvement

$\checkmark$ Reduced back pain

$\checkmark$ No influence on fracture healing 